\title{
Pteridófitas em fragmentos florestais da APA Fernão Dias, Minas Gerais, BrasiL ${ }^{1}$
}

\author{
Luciana Cláudia Neves Melo ${ }^{2,3}$ \& Alexandre Salino ${ }^{2}$
}

\section{Resumo}

(Pteridófitas em fragmentos florestais da APA Fernão Dias, Minas Gerais, Brasil) Foi realizado o estudo das pteridófitas ocorrentes na APA Fernão Dias, situada no extremo sul do estado de Minas Gerais, na Serra da Mantiqueira, abrangendo áreas dos municípios de Camanducaia, Gonçalves, Extrema e Itapeva. Esta região encontrase entre 1.000 e 2.068 metros de altitude, em zona de tensão ecológica entre as florestas tropicais montanas e submontanas. Constatou-se a ocorrência de 173 espécies de pteridófitas, distribuídas em 23 famílias e 55 gêneros. As famílias mais representativas foram Polypodiaceae (21 spp.), Thelypteridaceae (18 spp.), Lomariopsidaceae (17 spp.), Pteridaceae e Aspleniaceae (13 spp. cada), Blechnaceae e Hymenophyllaceae (12 spp. cada), Grammitidaceae (10 spp.) e Lycopodiaceae (8 spp.). Os gêneros com maior número de espécies foram Thelypteris e Elaphoglossum (17 spp. cada), Asplenium (13 spp.), Blechnum (12 spp.), Trichomanes (7 spp.), Campyloneurum (6 spp.), Pecluma, Polypodium, Huperzia e Hymenophyllum (5 spp. cada). A maioria das espécies é terrestre com distribuição neotropical. Athyrium filix-femina (L.) Roth. consiste na primeira referência para Minas Gerais. Este estudo revelou a presença de uma espécie ameaçada de extinção (Dicksonia sellowiana Hook.) e três presumivelmente ameaçadas (Alsophila capensis (L.f.) J. Sm., Dryopteris patula (Sw.) Underw. e Botrychium virginianum (L.) Sw.), constantes da Lista Vermelha das Espécies Ameaçadas de Extinção da Flora de Minas Gerais.

Palavras-chave: Pteridófitas, florística, floresta estacional semidecidual, floresta ombrófila.

\section{Abstract}

(Pteridophytes from APA Fernão Dias, Minas Gerais, Brazil) A floristic inventory of pteridophytes was carried out in the APA Fernão Dias, located in Serra da Mantiqueira, on the southern of Minas Gerais State, including the municipalities of Camanducaia, Gonçalves, Extrema and Itapeva. This region is situated between 1.000 and 2.068 meters of altitude, in a zone of Tropical seasonal semideciduous and Tropical rain forests. A total of 173 pteridophyte species were identified, distributed in 23 families and 55 genera. The most representative families were Polypodiaceae (21 spp.), Thelypteridaceae (18 spp.), Lomariopsidaceae (17 spp.), Pteridaceae and Aspleniaceae (13 spp. each), Blechnaceae and Hymenophyllaceae (12 spp. each), Grammitidaceae (10 spp.) and Lycopodiaceae (8 spp.). The genera with the highest numbers of species were Thelypteris and Elaphoglossum (17 spp. each), Asplenium (13 spp.), Blechnum (12 spp.), Trichomanes (7 spp.), Campyloneurum (6 spp.), Pecluma, Polypodium, Huperzia and Hymenophyllum (5 spp. each). Most of the species were terrestrial and has a Neotropical distribution. Athyrium filix-femina (L.) Roth. is the first record for the Minas Gerais State. This study has also identified the presence of a species threatened with extinction, Dicksonia sellowiana Hook., and of three species presumably threatened: Alsophila capensis (Sw.) Conant, Dryopteris patula (Sw.) Underw. and Botrychium virginianum (L.) Sw., included in the Red List of Threatened Species of the Flora of Minas Gerais State.

Key words: Pteridophytes, floristic, Tropical seasonal semideciduous forest, Tropical rain forest.

\section{INTRODUÇÃO}

As pteridófitas ocorrem nos mais variados ecossistemas, em uma grande variedade de ambientes: do nível do mar a elevadas altitudes, de regiões árticas-alpinas ao interior de florestas tropicais úmidas, de áreas subdesérticas no interior dos continentes até regiões rochosas costeiras e mangues (Page 1979). No entanto, sua maior diversidade encontra-se nos trópicos úmidos e nas montanhas subtropicais (Tryon \& Tryon 1982). Este grupo forma um importante componente da flora de florestas tropicais úmidas, compreendendo geralmente cerca de $10 \%$ do total do número de espécies de plantas vasculares (Grayum \& Churchill 1987; Melo $\&$ Salino 2002). Estima-se que haja entre 10.000 e 11.300 espécies de pteridófitas (Ross

Artigo recebido em 04/2006. Aceito para publicação em 01/2007.

${ }^{1}$ Parte da Dissertação de Mestrado da primeira autora desenvolvida no Curso de Pós-Graduação em Biologia Vegetal da Universidade Federal de Minas Gerais.

${ }^{2}$ Departamento de Botânica, Instituto de Ciências Biológicas, Universidade Federal de Minas Gerais, Cx. Postal 486, 30123-970, Belo Horizonte, MG, Brasil.

${ }^{3}$ Autor para correspondência: lucianamelo@ufmg.br 
1996). Conforme estimativa de Moran (1995a), há na América do Sul aproximadamente 3.000 espécies, sendo que no Brasil estima-se a ocorrência de 1.200 a 1.300 (Prado 1998) e Minas Gerais abriga pelo menos $50 \%$ desse total (Salino 2000).

Os estudos da pteridoflora no estado de Minas Gerais são fragmentados. Alguns trabalhos apresentam apenas listas de espécies (Brade 1942a, 1949; Lisboa 1954; Giulietti et al. 1987; Krieger \& Camargo 1990; Graçano et al. 1998; Melo \& Salino 2002 e Figueiredo \& Salino 2005). Há trabalhos florísticos que apresentam chaves de identificação, descrições e ilustrações, que são geralmente restritos a uma família, sendo quase todos realizados na Cadeia do Espinhaço (e.g. Carvalho 1982; Camargo 1987; Windisch \& Prado 1990; Prado 1992; Windisch 1992; Prado \& Windisch 1996; Prado 1997 e Prado \& Labiak 2003).

Os estudos para a região da Serra da Mantiqueira, onde se insere a área objeto deste trabalho, resumem-se aos de Brade (1937) na determinação de material colecionado durante uma excursão de Campos Porto à região de Campos do Jordão (SP); Brade (1949) relatório de excursão à Passa Quatro $(\mathrm{MG})$ e Brade (1942b) composição da flora de pteridófitas do Itatiaia.

No sul de Minas a cobertura vegetal original era constituída por extensas formações florestais (Azevedo 1962), que têm sido nas últimas décadas reduzida a fragmentos, que sofrem constantes pressões agropastoris. Este trabalho teve como objetivo documentar a riqueza de pteridófitas em formações florestais da Área de Proteção Ambiental (APA) Fernão Dias, Minas Gerais, subsidiando o conhecimento da composição florística da região sul do estado.

\section{Material e Métodos}

\section{Caracterização da área de estudo}

A Área de Proteção Ambiental (APA) Fernão Dias, criada como medida compensatória aos danos ambientais causados pela duplicação da BR 381 (Fernão Dias) situa-se no extremo sul do estado de Minas Gerais, na Serra da Mantiqueira e abrange seis municípios integralmente (Camanducaia, Extrema, Gonçalves, Itapeva, Sapucaí-Mirim e Toledo) e dois parcialmente (Paraisópolis e Brasópolis).

A APA inclui toda a bacia hidrográfica do rio Jaguari em Minas Gerais, da qual se destaca o rio Camanducaia como o maior e principal afluente. Formador do rio Piracicaba, já no estado de São Paulo, o rio Jaguari recebe em território paulista confluência de outros cursos d'água nascentes em Minas Gerais (DER/MG 1998).

A APA encontra-se entre 1.000 e 2.068 metros de altitude, em zona de tensão ecológica entre as formações de Floresta Ombrófila Densa, Ombrófila Mista e Estacional Semidecidual. Dependendo das condições climáticas, muitas vezes relacionadas a gradientes topográficos e ao relevo, predomina uma ou outra tipologia vegetal ou, em alguns casos, misturam-se aspectos de mais de um tipo (DER/MG 1998). Segundo a classificação de Köeppen (Ayoade 1998), o clima é temperado chuvoso (Cwb), com verão fresco. As temperaturas variam entre as médias de $14^{\circ} \mathrm{C}$ e $19^{\circ} \mathrm{C}$, podendo atingir, no inverno, temperaturas mínimas absolutas inferiores a $4^{\circ} \mathrm{C}$ (DER/MG 1998). No espaço geográfico da APA, o pluviômetro da fazenda Levantina registra as médias anuais de chuvas superiores a $1.500 \mathrm{~mm}$. Os excedentes hídricos ocorrem nos meses de outubro a abril, com a altura média anual das chuvas variando entre 1.600 a $1.800 \mathrm{~mm}$, e a evaporação potencial anual entre 650 e 800 mm (DER/MG 1998).

\section{Amostragem e análise dos dados}

As atividades de campo para coleta e registro dos dados foram desenvolvidas no período de dezembro de 2000 a fevereiro de 2002, realizando coletas nos diferentes períodos climáticos na APA Fernão Dias. Foram coletadas todas as pteridófitas através de 
caminhadas pelas bordas e interior de fragmentos florestais, em fundos de vale com cursos d'água, além de outros ambientes como barrancos de estradas, manchas ciliares e afloramentos rochosos, abrangendo áreas dos municípios de Camanducaia, Gonçalves, Extrema e Itapeva. Dados sobre o hábito das espécies foram registrados como epífita, hemiepífita, terrestre e rupícola. Para alguns táxons considerou-se como hábito variado aqueles que apresentaram mais de um hábito específico, muitas vezes acidental, i.e., epífita, rupícola e terrestre; rupícola e epífita; terrestre e epífita; hemiepífita, epífita e terrestre. As amostras foram coletadas e herborizadas segundo as técnicas usuais para as pteridófitas (Silva 1989). Os espécimes testemunhos foram depositados no Herbário do Departamento de Botânica, Instituto de Ciências Biológicas, Universidade Federal de Minas Gerais (BHCB) e duplicatas foram enviadas a especialistas. A identificação das espécies foi realizada com auxílio de literatura especializada e por comparação com material já determinado por especialistas existentes nos herbários BHCB, HB, R e RB (acrônimos segundo Holmgren et al. 1990).

Os táxons são apresentados em ordem alfabética de família de acordo com a Flora Mesoamericana (Moran 1995b). Para a família Vittariaceae utilizou-se a classificação genérica de Crane (1997). Para as abreviações dos nomes dos autores das espécies adotou-se Pichi-Sermolli (1996). O sistema de classificação da vegetação usado foi o de Veloso et al. (1991).

\section{Resultados e Discussão}

Foram encontradas nos fragmentos florestais, além de outros ambientes como barrancos de estradas e afloramentos rochosos, 173 espécies de pteridófitas, distribuídas em 23 famílias e 55 gêneros (Tab. 1). As famílias mais representativas foram Polypodiaceae com 21 espécies; Thelypteridaceae, com 18; Lomariopsidaceae, com 17; Pteridaceae e Aspleniaceae, com 13 cada; Blechnaceae e Hymenophyllaceae com 12 cada; Grammitidaceae com 10; e Lycopodiaceae, com oito espécies. Estas nove famílias representam $71,7 \%$ do total das espécies. Os gêneros com maior número de espécies foram Thelypteris e Elaphoglossum, com 17 cada; Asplenium, com 13; Blechnum, com 12; Trichomanes, com sete; Campyloneurum, com seis; Pecluma, Polypodium, Huperzia e Hymenophyllum, com cinco espécies cada. Estes 10 gêneros representam 53,2\% do total das espécies determinadas.

As pteridófitas nos fragmentos estudados são predominantemente herbáceas, mas também podem ser arborescentes a subarborescentes como Blechnum brasiliense. As arborescentes estão representadas pelos gêneros Cyathea, Sphaeropteris, Dicksonia e Alsophila. Dentre as herbáceas, $95(57,9 \%)$ são terrestres, $38(23,2 \%)$ epífitas, 18 (11\%) de hábito variado e $13(7,9 \%)$ rupícolas. Algumas espécies de hábito epífito, eventualmente ocorrem como terrestres na região estudada: Elaphoglossum erinaceum, E. tamandarei, E. burchellii, Pecluma recurvata, Asplenium auritum, A. harpeodes e A. wacketii. Como constatado por Dittrich et al. (2005), Blechnum binervatum ssp. acutum apresenta-se epífito no Parque Estadual do Pico do Marumbi e terrestre ou hemiepífito em outras áreas. Esses dados demonstram que este táxon possui hábito bastante variado, como constatado também na APA, onde se apresentou nos diferentes fragmentos como hemiepífito, epífito e terrestre.

A maioria das pteridófitas encontradas na área ocorre no interior das florestas, especialmente nas áreas mais úmidas, sendo que as espécies mais comuns foram: Asplenium harpeodes, A. auritum, Campyloneurum nitidum, Dicksonia sellowiana, Hypolepis rugulosa, Microgramma squamulosa, Pecluma pectinatiformis, Polypodium catharinae, $P$. hirsutissimum e Polystichum montevidense. Ao longo das áreas mais 
alteradas, principalmente nas margens das estradas e bordas de florestas, é comum a ocorrência de espécies como Blechnum occidentale, B. binervatum, Lycopodium clavatum, Dicranopteris flexuosa, Sticherus pruinosus e S. bifidus.

A especificidade quanto ao forófito foi observada apenas em algumas espécies como Pecluma truncorum e Trichomanes polypodioides que ocorrem apenas em caule de Cyatheaceae e Dicksoniaceae, como já ressaltado por Evans (1968) e Lellinger (1994). Muitas espécies coletadas foram observadas em locais com diferentes perturbações antrópicas, como Polypodium catharinae, $P$. hirsutissimum e Microgramma squamulosa, ao passo que outras de Hymenophyllaceae e Grammitidaceae foram observadas em áreas mais preservadas. Algumas espécies ocorrem preferencialmente em áreas bastante alteradas, como trilhas de gado e bordas de mata como Hypolepis rugulosa, que é abundante nestes ambientes, apesar de ter sido também registrada no interior de mata preservada. $\mathrm{Na}$ área estudada observa-se que Alsophila sternbergii ocorre com frequiência, em agrupamentos densos de indivíduos, principalmente, em ambientes alterados. Este padrão é citado por Tryon \& Tryon (1982) para a família Cyatheaceae, que pode formar populações densas em regiões montanhosas, sendo dominantes na vegetação. Padrão semelhante é observado em Alsophila setosa por Sylvestre \& Kurtz (1994), que apresentou importância moderada na estrutura da floresta, em função de sua elevada densidade relativa. Comentam ainda nesse estudo que A. setosa foi encontrada em agrupamentos de até 10 indivíduos, crescendo, especialmente, em formações secundárias. Schmitt \& Windisch (2005) também discutem o adensamento encontrado para A. setosa.

As espécies de Grammitidaceae geralmente ocorrem em áreas superiores a $1.000 \mathrm{~m}$, estando a ocorrência em altitudes inferiores restrita a táxons de ampla distribuição altitudinal, ou àqueles que ocorrem nos limites meridionais de distribuição da família (Labiak \& Prado 2003). Este padrão foi observado na área estudada, onde a família ocorre apenas nos fragmentos situados acima de $1.300 \mathrm{~m}$ de altitude. Estas observações de distribuição e arranjo das espécies foram feitas no campo, sem qualquer tipo de quantificação, porém refletem padrões já verificados em outros estudos (e.g. Dittrich et al. 2005; Poulsen 1996).

A ocorrência de espécies de pteridófitas epífitas na área estudada é consideravelmente baixa $(23,03 \%)$, quando comparada a outros estudos já realizados (e.g. Sylvestre 1997a e Dittrich et al. 2005). Geralmente as áreas de floresta atlântica da costa sudeste e sul do Brasil possuem grande quantidade de pteridófitas epífitas (Sylvestre 1997a; Dittrich et al. 2005 e Labiak \& Prado 1998). No entanto, dentre as áreas já inventariadas no estado de Minas Gerais, esta é a que registrou a maior ocorrência de epífitas. Figueiredo \& Salino (2005) registraram 26 (13,7\%) espécies epífitas em quatro Reservas Particulares do Patrimônio Natural (RPPN's) da Região Metropolitana de Belo Horizonte, Melo \& Salino (2002) registraram nove $(8,25 \%)$ epífitas no Parque Estadual do Rio Doce e oito $(8,42 \%)$ na Estação Biológica de Caratinga. A APA Fernão Dias revela uma mistura de elementos de florestas ombrófilas e de florestas estacionais, a qual poderia ser atribuído o baixo percentual de ocorrência de epífitas, já que aquelas semidecíduas contribuem com uma riqueza bem menor de espécies epifíticas.

Ressalta-se que os gêneros Huperzia, Elaphoglossum e Hymenophyllum e a família Grammitidaceae, que são comuns nas áreas de floresta atlântica litorânea, estão bem representados na área de estudo.

O número de espécies registradas na APA Fernão Dias (173) demonstra uma grande riqueza de pteridófitas, quando comparado a outras áreas estudadas (e.g. Sylvestre 1997a; Sylvestre 1997b; Salino \& Joly 2001; Melo \& Salino 2002; Prado \& Labiak 2003; Bueno \& Senna 1992; Brack et al. 1985; Paciencia \& 
Prado 2004). Se considerados os trabalhos similares publicados para o estado de Minas Gerais (Graçano et al. 1998; Melo \& Salino 2002; Figueiredo \& Salino 2005) a área da APA Fernão Dias apresentou a segunda maior riqueza de espécies de pteridófitas, contribuindo para o incremento de novos registros para o estado, além de abrigar espécies raras e ameaçadas de extinção.

No que se refere à distribuição geográfica das espécies, a grande maioria (57\%) é neotropical, corroborando com dados da literatura (Salino \& Joly 2001; Dittrich et al. 2005; Labiak \& Prado 1998), ou de ocorrência restrita ao Brasil (30\%). No que se refere às espécies brasileiras, $80,4 \%$ (41) são restritas às Regiões Sul e Sudeste; as outras 10 são de ampla distribuição. Constatou-se ainda que do total de espécies registradas na região, 27 possuem distribuição ampla nas Américas do Sul e Central; duas são paleotropicais: Macrothelypteris torresiana e Diplazium petersenii; e quatro são pantropicais: Lycopodiella cernua; Osmunda regalis; Cochlidium serrulatum e Pleopeltis macrocarpa. A maioria das espécies de Elaphoglossum apresentou distribuição restrita ao Brasil, exceto E. burchellii; E. gayanum; E. langsdorffii; E. ornatum e E. sellowianum.

O estudo na APA Fernão Dias apresentou importantes registros para a flora do estado, como Athyrium filix-femina, que apesar de ter distribuição ampla na América tropical, no Brasil é considerada rara, sendo aqui registrada como primeira referência para Minas Gerais. De acordo com Sehnem (1979) e Brade (1937), esta espécie ocorre no México, Peru, Colômbia e Brasil, nos estados do Rio de Janeiro, São Paulo, Santa Catarina e Rio Grande do Sul.

O levantamento florístico realizado em fragmentos da APA Fernão Dias revelou a presença de uma espécie ameaçada de extinção, Dicksonia sellowiana, e três presumivelmente ameaçadas, Alsophila capensis, Dryopteris patula e Botrychium virginianum, constantes da Lista Vermelha das Espécies Ameaçadas de Extinção da Flora de Minas Gerais (Mendonça \& Lins 2000).

Dicksonia sellowiana é um elemento característico da Floresta Ombrófila Mista e ocorre numa faixa de 1.600-2.300 m de altitude (Fernandes 1997). Na região, esta espécie se encontra bastante representada. As principais ameaças às espécies de pteridófitas na região são o desmatamento e alteração da cobertura vegetal, em especial pela agricultura, com conseqüente fragmentação e exposição das comunidades pteridofíticas a diferenças microclimáticas e edáficas, daquelas originais.

Alsophila capensis ocorre no sudeste do Brasil, em altitudes que variam entre 1.000 $2.000 \mathrm{~m}$, crescendo preferencialmente junto a rochas úmidas ou beira de córregos no interior das matas. Segundo Fernandes (1997), A. capensis é citada como espécie típica da região de Araucárias no sul do Brasil e nos estados de Minas Gerais, São Paulo e Rio de Janeiro, em formações cuja ocorrência está associada a Dicksonia sellowiana e Araucaria angustifolia (Bertol.) Kuntze, espécies típicas da Floresta Ombrófila Mista. Dryopteris patula ocorre desde o México, Antilhas até sul da Argentina e no sudeste do Brasil. Apesar de sua ampla distribuição nas Américas Central e Sul, no Brasil ela é bastante restrita, com ocorrência apenas no Mato Grosso e Minas Gerais. Ressalta-se, que em Minas Gerais somente se tem conhecimento de coletas na Serra da Piedade e em um fragmento da APA Fernão Dias. Dessa forma, foi incluída como uma espécie presumivelmente ameaçada de extinção, com suas populações expostas a alta pressão dos desmatamentos e ações humanas. Botrychium virginianum é uma espécie rara, única representante do gênero no Brasil, ocorrendo em poucos fragmentos da região. No Brasil, $B$. virginianum ocorre nos estados de Santa Catarina, Rio de Janeiro e Minas Gerais. Em Minas Gerais esta espécie está registrada apenas para a região da Serra da Mantiqueira. 
Muito embora alguns fragmentos florestais aqui estudados estejam em bom estado de conservação, a área da APA está sujeita a um elevado grau de pressão para formação de pastos e lavouras. Diversas espécies ainda resistem à movimentação antrópica e outras sucumbem à degradação. As particularidades da região da APA Fernão Dias, com uma elevada riqueza de pteridófitas (173) ocorrentes em poucos fragmentos florestais, corroboram com esforços de implantação de políticas de preservação e do plano de manejo da APA, possibilitando a manutenção da preservação dessa diversidade remanescente.

Tabela 1 - Lista das espécies de pteridófitas ocorrentes na APA Fernão Dias, Minas Gerais, Brasil. EP: epífita; HE: hemiepífita; RU: rupícola; TR: terrestre.

\begin{tabular}{|c|c|c|}
\hline Taxa & Hábito & Material examinado \\
\hline \multicolumn{3}{|l|}{ ASPLENIACEAE } \\
\hline Asplenium abscissum Willd. & $\mathrm{TR}$ & Mota 1285 \\
\hline A. auriculatum Sw. & $\mathrm{EP} / \mathrm{RU}$ & Melo 148; Salino 5611, 5769, 6406, 6927 \\
\hline A. auritum $\mathrm{Sw}$. & $\mathrm{EP} / \mathrm{RU} / \mathrm{TR}$ & $\begin{array}{l}\text { Melo } 125,145 \text {; Salino 5580, 5785, 6869, 6883, } \\
6407 \text {; Mota 1283; Torres } 1242\end{array}$ \\
\hline A. claussenii Hieron. & $\mathrm{TR}$ & Melo 212; Salino 5625, 6394 \\
\hline A. harpeodes Kunze & $\mathrm{EP} / \mathrm{TR}$ & $\begin{array}{l}\text { Melo 32, 74, 115; Salino 5573, 5774, 5620, } \\
5626,6393\end{array}$ \\
\hline A. incurvatum Fée & $\mathrm{EP} / \mathrm{RU}$ & Melo 60; Salino 6415, 6936; Mota 1284 \\
\hline A. kunzeanum Klotzsch ex Rosenst. & $\mathrm{TR}$ & Salino 4930 \\
\hline A. scandicinum Kaulf. & $\mathrm{EP}$ & Melo 192 \\
\hline A. praemorsum Sw. var. praemorsum & $\mathrm{EP}$ & Melo 187 \\
\hline A. radicans var. uniseriale (Raddi) L.D. Gómez & $\mathrm{TR}$ & Melo 117, 173 ; Salino 6429 \\
\hline A. serra Langsd. \& Fisch. & RU & $\begin{array}{l}\text { Salino 5600, 5770, 6411, 6937; Melo 33; } \\
\text { Meireles } 419\end{array}$ \\
\hline A. triquetrum N. Murak. \& R.C. Moran & RU & Melo 119 \\
\hline A. wacketii Rosenst. & $\mathrm{EP} / \mathrm{TR}$ & Melo 44, 166; Salino 6414, 6939 \\
\hline \multicolumn{3}{|l|}{ BLECHNACEAE } \\
\hline Blechnum austrobrasilianum de la Sota & $\mathrm{TR}$ & Melo 213, 105; Salino 5632 \\
\hline $\begin{array}{l}\text { B. binervatum ssp. acutum (Desv.) } \\
\text { R.M. Tryon \& Stolze }\end{array}$ & $\mathrm{HE} / \mathrm{EP} / \mathrm{TR}$ & $\begin{array}{l}\text { Melo 141, 169, 208, 211; Salino 5780, 5790, } \\
6917\end{array}$ \\
\hline B. brasiliense Desv. & TR & Salino 6891 \\
\hline B. $\mathrm{x}$ caudatum Cav. & $\mathrm{TR}$ & Melo 97, 197 \\
\hline B. cordatum (Desv.) Hieron. & $\mathrm{TR}$ & Melo 79, 203, 142; Salino 6896 \\
\hline B. gracile Kaulf. & $\mathrm{TR}$ & Melo 207; Salino 6898 \\
\hline B. occidentale L. & $\mathrm{TR}$ & Salino 5568, 6905 \\
\hline B. polypodioides Raddi & $\mathrm{TR}$ & Melo 85; Salino 6904, 6886, 5639 \\
\hline B. pteropus (Kunze) Mett. & TR & Melo 169; Salino 5780, 6917 \\
\hline B. schomburkgii Klotzsch & $\mathrm{TR}$ & Melo 75; Salino 6416 \\
\hline B. spannagelii Rosenst. & $\mathrm{TR}$ & Melo 78, 99; Salino 6397, 5623 \\
\hline B. usterianum (Christ) C. Chr. & $\mathrm{TR}$ & Melo 111; Salino 5627 \\
\hline \multicolumn{3}{|l|}{ CYATHEACEAE } \\
\hline $\begin{array}{l}\text { Alsophila capensis subsp. polypodioides } \\
\text { (Sw.) D.S. Conant }\end{array}$ & TR & Melo 116, 171; Meireles 421 \\
\hline
\end{tabular}




\begin{tabular}{|c|c|c|}
\hline Taxa & Hábito & Material examinado \\
\hline A. setosa Kaulf. & TR & Melo 172; Salino 5624, 5778, 6427 \\
\hline A. sternbergii (Sternb.) D.S. Conant & TR & Melo 177 \\
\hline Cyathea corcovadensis (Raddi) Domin & TR & Salino 5599, 6430, 6916 \\
\hline C. delgadii Sternb. & TR & Salino 6873 \\
\hline C. phalerata Mart. & TR & Melo 120 \\
\hline Sphaeropteris gardneri (Hook.) R.M. Tryon & TR & Salino 6893 \\
\hline \multicolumn{3}{|l|}{ DENNSTAEDTIACEAE } \\
\hline Dennstaedtia dissecta (Sw.) T. Moore & TR & Melo 112, 183; Salino 5612 \\
\hline D. globulifera (Poir.) Hieron. & TR & Melo 202 \\
\hline Hypolepis rugulosa (Labill.) J. Sm. & TR & Salino 5596, 6432 \\
\hline Histiopteris incisa (Thunb.) J. Sm. & TR & Salino 5619, 6396 \\
\hline Lindsaea stricta (Sw.) Dryand. & TR & Salino 6887 \\
\hline Pteridium arachnoideum (Kaulf.) Maxon & TR & Salino 6900 \\
\hline \multicolumn{3}{|l|}{ DICKSONIACEAE } \\
\hline Dicksonia sellowiana Hook. & TR & Melo 70; Salino 5598, 5622, 6874 \\
\hline \multicolumn{3}{|l|}{ DRYOPTERIDACEAE } \\
\hline Dryopteris patula (Sw.) Underw. & TR & Melo 86 \\
\hline D. wallichiana (Spring) Hyl. & TR & Melo 72; Meireles 368; Salino 5610, 5783, 6940 \\
\hline Polystichum montevidense (Spreng.) Rosenst. & TR & $\begin{array}{l}\text { Melo 31, 101; Mota 1292; Salino 5569, 5576, } \\
6878\end{array}$ \\
\hline Rumohra adiantiformis (Forst.) Ching & TR & Melo 66; Salino 6880 \\
\hline \multicolumn{3}{|l|}{ GLEICHENIACEAE } \\
\hline Dicranopteris flexuosa (Schrad.) Underw. & TR & Salino 5556, 6889, 6921 \\
\hline D. nervosa (Kaulf.) Ching & TR & Melo 135; Salino 6399, 5641 \\
\hline Sticherus bifidus (Willd.) Ching & TR & Melo 80; Salino 6890 \\
\hline S. pruinosus (Mart.) Ching & TR & Salino 6400, 6903 \\
\hline S. lanuginosus (Fée) Nakai & TR & Salino 5640, 6892 \\
\hline S. revolutus (Kunth) Ching & TR & Salino 6913 \\
\hline \multicolumn{3}{|l|}{ GRAMMITIDACEAE } \\
\hline Ceradenia albidula (Baker) L.E. Bishop & $\mathrm{EP}$ & Melo 154 \\
\hline Cochlidium punctatum (Raddi) L.E. Bishop & $\mathrm{EP} / \mathrm{RU}$ & Melo 47, 88; Salino 5638 \\
\hline C. serrulatum (Sw.) L.E. Bishop & $\mathrm{EP}$ & Mota 1305 \\
\hline Grammitis fluminensis Fée & EP & Melo 94 \\
\hline $\begin{array}{l}\text { Lellingeria apiculata (Kunze ex Klotszch) } \\
\text { A.R. Sm. \& R.C. Moran }\end{array}$ & TR & Mota 1304 \\
\hline L. organensis (Gardner) A.R. Sm. \& R.C. Moran & $\mathrm{EP}$ & Melo 24; Salino 5607, 6405, 6933 \\
\hline $\begin{array}{l}\text { Melpomene flabelliformis (Poir.) A.R. Sm. \& } \\
\text { R.C. Moran }\end{array}$ & $\mathrm{EP}$ & Melo 89 \\
\hline M. peruviana (Desv.) A.R. Sm. \& R.C. Moran & RU & Salino 5637 \\
\hline $\begin{array}{l}\text { M. pilosissima (M. Martens \& Galeotti) } \\
\text { A.R. Sm. \& R.C. Moran }\end{array}$ & EP & Melo 23, 67 \\
\hline Terpsichore gradata (Baker) A.R. Sm. & $\mathrm{EP} / \mathrm{RU}$ & Melo 35; Salino 6934 \\
\hline
\end{tabular}




\begin{tabular}{|c|c|c|}
\hline Taxa & Hábito & Material examinado \\
\hline \multicolumn{3}{|l|}{ HYMENOPHYLLACEAE } \\
\hline Hymenophyllum caudiculatum Mart. & TR & Melo 134 \\
\hline H. elegans Spreng. & RU & Melo 162 \\
\hline H. fucoides Sw. & $\mathrm{EP}$ & Melo 90 \\
\hline H. polyanthos (Sw.) Sw. & $\mathrm{EP}$ & Melo55, 155; Salino 5606,6423, 6424; Mota 1286 \\
\hline H. rufum Fée & $\mathrm{EP}$ & Melo 54, 144; Salino 6422; 6930 \\
\hline Trichomanes anadromum Rosenst. & $\mathrm{EP}$ & Melo 127; Salino 6870 \\
\hline T. angustatum Carm. & $\mathrm{EP}$ & Melo 130; Salino 5592, 6863 \\
\hline T. diaphanum Humb. \& Bonpl. ex Kunth & $\mathrm{EP}$ & Salino 6914 \\
\hline T. hymenoides L. & $\mathrm{EP}$ & Melo 131 \\
\hline T. polypodioides L. & $\mathrm{EP}$ & Mota 1303 \\
\hline T. reptans $\mathrm{Sw}$. & RU & Salino 6420, 6931 \\
\hline T. rigidum $\mathrm{Sw}$. & TR & Salino 6428 \\
\hline
\end{tabular}

\section{LOMARIOPSIDACEAE}

Elaphoglossum acrocarpum (Mart.) T. Moore

E. eximium (Mett.) Christ

TR Meireles 420

E. blanchetii (Mett.) C. Chr.

Melo 69; Salino 6410, 5618

E. burchellii (Baker) Christ

Mota 1281

E. erinaceum (Fée) T. Moore

Melo 113, 139; Salino 6871

E. gayanum (Fée) T. Moore

EP/TR

Melo 140, 156

E. hymenodiastrum (Fée) Brade

RU/TR

Melo 73, 68, 147, 176; Salino 6431

E. langsdorffii (Hook. \& Grev.) T. Moore

Melo 193

E. macahense (Fée) Rosenst.

Mota 1302

TR

Melo157

E. minutum (Pohl ex Fée) T. Moore

Melo 143

E. organense Brade

Salino 6410b

E. ornatum (Mett.) Christ

Melo 137

E. pachydermum (Fée) T. Moore

Melo 30, 138

E. paulistanum Rosenst.

Melo 168

E. sellowianum (Kaulf.) T. Moore

Salino 5604

E. tamandarei Brade

Salino 6872

E. vagans (Mett.) Hieron.

Melo 29, 71, 185; Salino 5605, 5617, 6402; Mota 1282; Torres 1146, 1241

\section{LOPHOSORIACEAE}

Lophosoria quadripinnata (G. F. Gmel.) C. Chr. TR

Melo 133; Mota 1306

\section{LYCOPODIACEAE}

Huperzia biformis (Hook.) Holub.

RU/EP

Melo 91; Salino 5767, 5609; Meireles 508

H. christii (Silveira) Holub.

RU

Melo 63

H. pungentifolia (Silveira) B. Øllg.

EP Salino 5784

H. hexasticha B. Øllg. \& P.G. Windisch

EP/RU

Melo 62, 87, 132; Meireles 507; Salino 5575, 5616

H. reflexa (Lam.) Trevis.

TR Melo 82; Salino 5590, 6944

Lycopodiella cernua (L.) Pic.Serm.

TR

Melo 84; Mota 1291; Salino 6909 


\begin{tabular}{lll}
\hline Taxa & Hábito & Material examinado \\
\hline Lycopodium clavatum L. & TR & Melo 41; Salino 5571, 6918 \\
L. thyoides Willd. & TR & Melo 83; Salino 5630
\end{tabular}

\section{MARATTIACEAE}

Marattia laevis J. Sm.

\section{OSMUNDACEAE}

Osmunda regalis L.

\section{OPHIOGLOSSACEAE}

Botrychium virginianum (L.) Sw.

\section{POLYPODIACEAE}

Campyloneurum acrocarpon Fée

C. aglaolepis (Alston) de la Sota

C. cf. angustifolium (Sw.) Fée

C. austrobrasilianum (Alston) de la Sota

C. minus Fée

C. nitidum (Kaulf.) C. Presl

Microgramma squamulosa (Kaulf.) de la Sota

Niphidium crassifolium (L.) Lellinger

Pecluma pectinatiformis (Lindm.) M.G. Price

P. robusta (Fée) M. Kessler \& A.R. Sm.

P. recurvata (Kaulf.) M.G. Price

$P$. singerii (de la Sota) M.G. Price

P. truncorum (Lindm.) M.G. Price

Phlebodium pseudoaureum (Cav.) Lellinger

Pleopeltis astrolepis (Liebm.) Fourn.

P. macrocarpa (Willd.) Kaulf.

Polypodium catharinae Langsd. \& Fisch.

P. hirsutissimum Raddi

P. latipes Langsd. \& Fisch

P. pleopeltidis Fée

$P$. vacillans Link

\section{PTERIDACEAE}

Adiantopsis chlorophylla (Sw.) Fée

A. radiata (L.) Fée

A. regularis (Kunze) T. Moore

Adiantum lorentzii Hieron.

A. raddianum $\mathrm{C}$. Presl.

A. subcordatum $\mathrm{Sw}$.

Doryopteris crenulans (Fée) Christ

D. nobilis (T. Moore) C. Chr.
TR

Melo 98; Salino 6398, 6941

TR

Melo 95

TR

Melo 52; Salino 5629

$\begin{array}{ll}\text { EP } & \text { Salino 5490, 5773 } \\ \text { EP } & \text { Salino 5589, 6895 } \\ \text { EP } & \text { Melo 186 } \\ \text { EP } & \text { Melo 191; Salino 5781, 5779; Mota } 1288 \\ \text { RU } & \text { Melo 209 } \\ \text { EP/RU } & \text { Melo 36, 59; Salino 5582, 5583, 6865, 6926; } \\ & \text { Mota 1295; Torres 1238; Meireles 412 } \\ \text { EP } & \text { Melo 37, 122; Salino 5782, 5570, 6899; } \\ \text { EP } & \text { Melo 206; Mota 1301 } \\ \text { EP } & \text { Melo 25, 121, 150; Mota 1297; Salino 5574 } \\ \text { EP } & \text { Melo 108 } \\ \text { EP/RU/TR } & \text { Melo 107, 151; Salino 5594, 5776, 6413, 6907 } \\ \text { EP } & \text { Mota 1299 } \\ \text { EP } & \text { Melo 126; Salino 6938 } \\ \text { TR } & \text { Melo 178 } \\ \text { EP } & \text { Salino 6881 } \\ \text { EP } & \text { Melo 57, 149; Salino 5608, 6418 } \\ \text { EP } & \text { Melo 27, 77, 136; Mota 1296; Salino 5480, } \\ & \text { 5636, 6419, 6876; Torres 1107 } \\ \text { EP } & \text { Melo 21, 64; Salino 5581, 6879 } \\ \text { TR } & \text { Melo 163, 165, 181, 184 } \\ \text { EP } & \text { Melo 28, 146; Salino 5587, 5593 } \\ \text { TR } & \text { Melo 190 }\end{array}$

TR

Melo 65, 114; Salino 6877

Melo 161; Salino 6882

Melo 46

Melo 205; Salino 6901

Melo 92, 123, 201; Mota 1298

Salino 6908

Melo 68; Salino 6902

Mota 1300 


\begin{tabular}{|c|c|c|}
\hline Taxa & Hábito & Material examinado \\
\hline Eriosorus myriophyllus (Sw.) Copel. & TR & Melo 20; Salino 6392, 6920 \\
\hline Pteris decurrens $\mathrm{C}$. Presl & TR & Salino 5635 \\
\hline P. deflexa Link & TR & Melo 110, 96; Salino 6403, 5771 \\
\hline P. lechleri Mett. & TR & Melo 159,167 \\
\hline P. splendens Kaulf. & TR & Melo 158, 194 \\
\hline \multicolumn{3}{|l|}{ SCHIZAEACEAE } \\
\hline Anemia imbricata Sturm. & TR & Mota 1287 \\
\hline A. phyllitidis (L.) Sw. & TR & Salino 5621, 6884; Torres 1305 \\
\hline A. raddiana Link & TR & Melo 128, 199 \\
\hline A. warmingii Prantl & TR & Melo 160 \\
\hline \multicolumn{3}{|l|}{ SELAGINELLACEAE } \\
\hline $\begin{array}{l}\text { Selaginella marginata (Humb. \& Bonpl. } \\
\text { ex Willd.) Spring }\end{array}$ & TR & Melo 129 \\
\hline S. muscosa Spring & TR & Melo 188; Salino 6425, 6875, 6928; Mota 1290 \\
\hline S. tenuissima Fée & RU & Melo 93 \\
\hline
\end{tabular}

\section{TECTARIACEAE}

Ctenitis submarginalis (Langsd. \& Fisch.) Ching TR Lastreopsis amplíssima (C. Presl) Tindale TR

L. effusa (Sw.) Tindale

Megalastrum crenulans (Fée) A.R. Sm. \& TR

R.C. Moran

M. umbrinum (C. Chr) A.R. Sm. \& R.C. Moran TR

Megalastrum sp.

\section{THELYPTERIDACEAE}

Macrothelypteris torresiana (Gaudich.) Ching TR Thelypteris amambayensis (Christ) Ponce TR

T. araucariensis Ponce

T. conspersa (Schrad.) A.R. Sm.

T. decurtata (Link) de la Sota

T. eriosora (Fée) Ponce

T. lugubris (Kunze ex Mett.) Ponce

T. metteniana Ching

T. oligocarpa (Humb. \& Bonpl. ex Willd.) Ching TR

T. opposita (Vahl) Ching

T. pachyrhachis (Mett.) Ching

T. ptarmica (Mett.) C.F. Reed

T. patens (Sw.) Small

T. raddii (Rosenst.) Ponce

T. retusa (Sw.) C.F. Reed

T. riograndensis (Lindm.) C.F. Reed

T. tamandarei (Rosenst.) Ponce
Melo 102; Salino 5572, 6885

Salino 5631, 6395

Melo 196

Melo 174; Salino 5615

Melo 210; Salino 6932

Melo 124

Salino 6897

Melo 106; Mota 1289; Salino 5585, 6911, 6924

Salino 6922

Melo 200; Salino 5586

Salino 5584

Melo 34,179; Salino 5601, 6942

Melo 189; Salino 5628, 6888

Salino 6925

Melo 40, Salino 6935

Melo 81; Salino 5578

Melo 103; Salino 5602

Salino 6915

Salino 6912

Melo 39, 76; Salino 5577, 5579, 5597, 5614, $5633,5765,6866,6867,6868$

Melo 49, 109; Salino 6919

Salino 6910

Salino 5613 


\begin{tabular}{|c|c|c|}
\hline Taxa & Hábito & Material examinado \\
\hline T. tenerrima (Fée) C.F. Reed & TR & Salino 6417 \\
\hline \multicolumn{3}{|l|}{ VITTARIACEAE } \\
\hline Polytaenium lineatum (Sw.) J. Sm. & $\mathrm{EP} / \mathrm{RU}$ & $\begin{array}{l}\text { Melo 22, 104; Salino 5588, 5777, 6404, 6906, } \\
6923,6929\end{array}$ \\
\hline Vittaria graminifolia Kaulf. & $\mathrm{EP}$ & Melo45, 153,152; Salino 5591,5791,5768,6408b \\
\hline V. lineata (L.) J. Sm. & EP & Melo 53,58; Salino 5786,5764, 6408; Mota 1293 \\
\hline \multicolumn{3}{|l|}{ WOODSIACEAE } \\
\hline Athyrium filix-femina (L.) Roth. & TR & Salino $6412,5766,5789$ \\
\hline Diplazium leptocarpon Fée & TR & Melo 100 \\
\hline D. lindbergii (Mett.) Christ & TR & Melo 175 \\
\hline D. petersenii (Kunze) Christ & TR & Melo 198; Salino 6894 \\
\hline D. turgidum Rosenst. & TR & Melo 118, 182; Salino 6943 \\
\hline
\end{tabular}

\section{Agradecimentos}

Agradecemos ao MMA/PROBIO pela concessão de bolsa e equipamento para os trabalhos de campo. À Prof ${ }^{a}$. Dra. Edivani Villaron Franceschinelli, coordenadora do PROBIO/Camanducaia. À Coordenação de Aperfeiçoamento de Pessoal de Nível Superior (CAPES) pela concessão de bolsa de Mestrado. Aos proprietários das matas estudadas, por nos permitirem caminhar e coletar os dados necessários. Ao Toninho, João Batista, Fernanda Carvalho, Zé Maria, Sueli, Cristina, Lia Teixeira, Noella Markstein, Lionel Van Rees, Ana Oliveira e Felipe Leite pelo auxílio nos trabalhos de campo. Ao Prof. Dr. Júlio Lombardi pela colaboração no herbário BHCB, e ao pteridólogo Vinícius Dittrich pela identificação de material botânico e auxílio nos trabalhos de campo. Aos revisores e editores pelas importantes sugestões para a melhoria do manuscrito.

\section{REFERÊNCIAS BibliográFICAS}

Azevedo, L. G. 1962. Tipos de vegetação do sul de Minas e campos da Mantiqueira (Brasil). Anais da Academia Brasileira de Ciências 34(2).
Ayoade, J. O. 1998. Introdução a climatologia para os trópicos. $5^{\mathrm{a}}$ ed. Bertrand Brasil, Rio de Janeiro, 332p.

Brack, P.; Bueno, R. M.; Flakenberg, D. B.; Paiva, M. R. C.; Sobral, M. \& Stehmann, J. R. 1985. Levantamento florístico do Parque Estadual do Turvo, Tenente Portela, Rio Grande do Sul, Brasil. Roessléria 7: 69-94.

Brade, A. C. 1937. Pteridophytas coletadas em Campos do Jordão, em 1937 pelo Dr. Campos Porto e determinadas por A. C. Brade. Rodriguésia 9: 113-116. 1942a. Excursão à Serra do Caparaó. Rodriguésia 15: 87-92. 1942b. A composição da flora pteridofítica do Itatiaia. Rodriguésia 6(15): 29-43.

1949. Relatório de uma excursão ao município de Passa Quatro, estado de Minas Gerais. Rodriguésia 23: 133-142.

Bueno, R. M. \& Senna, R. M. 1992. Pteridófitas do Parque Nacional dos Aparados da Serra I. Região do Paradouro. Caderno de Pesquisa Serie Botânica 4(1): 5-12.

Camargo, R. F. N. 1987. Pteridófitas rupícolas e saxícolas do sudeste de Minas Gerais 
(Brasil). Dissertação de Mestrado. Universidade Federal do Rio de Janeiro, Rio de Janeiro.

Carvalho, I. R. 1982. O gênero Anemia Sw. nos campos rupestres da Cadeia do Espinhaço no estado de Minas Gerais. Dissertação de Mestrado. Universidade Estadual de São Paulo, Rio Claro.

Crane, E. H. 1997. A revised circumscription of the genera of the fern family Vittariaceae. Systematic Botany 22(3): 509-517.

DER/MG. 1998. APA Fernão Dias. Plano de Gestão Ambiental. Vol.1. Belo Horizonte, 225p.

Dittrich, V. A. O.; Waechter, J. L. \& Salino, A. 2005. Species richness of pteridophytes in a montane Atlantic rain forest plot of southern Brazil. Acta Botanica Brasilica 19(3): 519-525.

Evans, A. M. 1968. Interspecific relationships in the Polypodium pectinatum-plumula complex. Annals of the Missouri Botanical Garden 55(3): 193-293.

Fernandes, I. 1997. Taxonomia e fitogeografia de Cyatheaceae e Dicksoniaceae nas Regiões Sul e Sudeste do Brasil. Tese de Doutorado. Universidade de São Paulo, São Paulo.

Figueiredo, J. B. \& Salino, A. 2005. Pteridófitas de quatro reservas particulares do patrimônio natural ao sul da região metropolitana de Belo Horizonte, Minas Gerais, Brasil. Lundiana 6(2): 83-94.

Giulietti, A. M.; Menezes, N. L.; Pirani, J. R.; Meguro, M. \& Wanderley, M. G. L. 1987. Flora da Serra do Cipó, Minas Gerais: Caracterização e lista de espécies. Boletim de Botânica da Universidade de São Paulo 9: 1-151.

Graçano, D.; Prado, J. \& Azevedo, A. A. 1998. Levantamento preliminar de Pteridophyta do Parque Estadual do Rio Doce (MG). Acta Botanica Brasilica 12(2): 165-181.
Grayum, M. H. \& Churchill, H. W. 1987. An introduction to the pteridophyte flora of Finca La Selva, Costa Rica. American Fern Journal 77: 73-89.

Holmgren, P. K.; Holmgren, N. H. \& Barnett, L. 1990. Index herbariorum. Part 1: The Herbaria of the world. 8ed. New York Botanical Garden, New York, 693p.

Krieger, L. \& Camargo, R. F. N. 1990. Pteridófitos da Zona da Mata de Minas Gerais encontrados no Herbário da Universidade Federal de Juiz de Fora. Anais do $36^{\circ}$ Congresso Nacional de Botânica, Curitiba. Pp. 287-306.

Labiak, P. H. \& Prado, J. 1998. Pteridófitas epífitas da Reserva Volta Velha, Itapoá Santa Catarina, Brasil. Boletim do Instituto de Botanica 11: 1-79.

Labiak, P. H. \& Prado, J. 2003. Grammitidaceae (Pteridophyta) no Brasil, com ênfase nos gêneros Ceradenia, Cochlidium e Grammitis. Hoehnea 30(3): 243-283.

Lellinger, D. B. 1994. Trichomanes polypodioides and its allies. American Fern Journal 81(1): 24-37.

Lisboa, M. A. 1954. Pteridophytes de Ouro Preto. Anais da Escola de Minas de Ouro Preto 29: 21-76.

Melo, L. C. N. \& Salino, A. 2002. Pteridófitas de duas áreas de floresta da bacia do Rio Doce no estado de Minas Gerais. Lundiana 3(2): 129-139.

Mendonça, M. P. \& Lins, L. V. (orgs.). 2000. Lista Vermelha das Espécies Ameaçadas de Extinção da Flora de Minas Gerias. Biodiversitas \& Fundação Zôo-Botânica de Belo Horizonte, Belo Horizonte, 157p.

Moran, R. C. 1995a. The importance of mountains to pteridophytes, with emphasis on neotropical montane Forests. In: Churchill, S. P.; Balslev, H.; Forero, E. \& Luteyn, J. L. (eds.). Biodiversity and Conservation of Neotropical Montane Forests. The New York Botanical Garden, New York. Pp. 359-363. 
1995b. Clave para las familias de Pteridofitas. In: Davidse, G.; Souza, M. S. \& Knapp, S. (eds.). Flora Mesoamericana. Vol. 1. Psilotaceae a Salvinaceae. Universidad Nacional Autónoma de México, México, D.F. Pp. 1-2

Paciencia, M. L. B. \& Prado, J. 2004. Efeitos de borda sobre a comunidade de pteridófitas na Mata Atlântica da Região de Una, sul da Bahia, Brasil. Revista Brasileira de Botânica 27(4): 641-653.

Page, C. N. 1979. The diversity of ferns. An ecological perspective. In: Dyer, A. F. (ed.). The experimental biology of the ferns. Academic Press, London. Pp. 10-56.

Pichi-Sermolli, R. E. G. 1996. Authors of scientific names in Pteridophyta. Royal Botanic Gardens, Kew, 78p.

Poulsen, A. D. 1996. Species richness and density of ground herbs within a plot of lowland rainforest in north-west Borneo. Journal Tropical of Ecology 12(2): 177-190.

Prado, J. 1992. Flora da Serra do Cipó, Minas Gerais. Pteridaceae-Cheilanthoideae. Boletim de Botânica da Universidade de São Paulo 13: 141-159.

1997. Flora da Serra do Cipó, Minas Gerais: Pteridaceae - Adiantoideae e Taenitoideae. Boletim de Botânica da Universidade de São Paulo 16: 115-118. 1998. Pteridófitas do Estado de São Paulo. In: Bicudo, C. E. M. \& Shepherd, G. J. (eds.). Biodiversidade do Estado de São Paulo: Síntese do Conhecimento ao Final do século XX - Fungos Macroscópicos e Plantas. FAPESP, São Paulo. Pp. 49-61.

Prado, J. \& Labiak, P. H. 2003. Flora de Grão Mogol, Minas Gerais: Pteridófitas. Boletim de Botânica da Universidade de São Paulo 21: 25-47.

Prado, J. \& Windisch, P. G. 1996. Flora da Serra do Cipó, Minas Gerais: Dennstaedtiaceae. Boletim de Botânica da Universidade de São Paulo 15: 83-88.
Ross, M. 1996. Mapping the world's pteridophyte diversity - systematics and floras. In: Camus, J. M.; Gibby, M. \& Johns, R. J. (eds.). Pteridology in Perspective. Royal Botanic Gardens, Kew. Pp. 29-42.

Salino, A. 2000. Pteridófitas. In: Mendonça, M. P. \& Lins, L. V. (orgs.). Lista Vermelha das Espécies Ameaçadas de Extinção da Flora de Minas Gerais. Biodiversitas \& Fundação Zoo-Botânica de Belo Horizonte, Belo Horizonte. Pp. 113-157.

Salino, A. \& Joly, C. A. 2001. Pteridophytes of Three remnants of gallery forest in the Jacaré-Pepira River Basin, São Paulo State, Brazil. Boletim Herbário Ezechias Paulo Heringer 8: 5-15.

Schmitt, J. L. \& Windisch, P. G. 2005. Aspectos ecológicos da Alsophila setosa Kaulf. (Cyatheaceae, Pteridophyta) no Rio Grande do Sul, Brasil. Acta Botânica Brasilica 19(4): 859-865.

Sehnem, A. 1979. Aspidiáceas. In: Reitz, R. (ed.). Flora Ilustrada Catarinense. Herbario Barbosa Rodrigues, Itajaí.

Silva, A. T. 1989. Pteridófitas. In: Bovoni, V. L. R. \& Fidalgo, O. (eds.). Técnicas de coleta, preservação e herborização de material botânico. Instituto de Botânica, São Paulo. Pp. 33-34

Sylvestre, L. S. 1997a. Pteridófitas da Reserva Ecológica de Macaé de Cima. In: Lima, H. C. \& Guedes-Bruni, R. R. (orgs.). Serra de Macaé de Cima: diversidade florística e conservação em Mata Atlântica. Ministério do Meio Ambiente e da Amazônia Legal/IBAMA. Jardim Botânico do Rio de Janeiro, Rio de Janeiro. Pp. 41-52.

1997b. Pteridophyta. In: Marques, M. C. M.; Vaz, A. S. F. \& Marquete, R. (eds.). Mapeamento da cobertura vegetal e listagem das espécies ocorrentes na Área de Proteção Ambiental de Cairuçu, Município de Parati, Rio de Janeiro. Jardim 
Botânico do Rio de Janeiro/Ministério do Meio Ambiente, Rio de Janeiro. Pp. 44-49. Sylvestre, L. S. \& Kurtz, B. C. 1994. Cyatheaceae. In: Lima, M. P. M. \& Guedes-Bruni, R. R. (orgs.). Reserva Ecológica de Macaé de Cima, Nova Friburgo-RJ. Aspectos florísticos das espécies vasculares. Vol.1. Ministério do Meio Ambiente e da Amazônia Legal/ IBAMA. Jardim Botânico do Rio de Janeiro, Rio de Janeiro. Pp. 139-152.

Tryon, R. M. \& Tryon, A. F. 1982. Ferns and allied plants, with special reference to tropical America. Springer Verlag, 857p.
Veloso, H. P.; Rangel Filho, A. L. \& Lima, J. C. 1991. Classificação da vegetação brasileira, adaptada a um sistema universal. IBGE, Departamento de Recursos Naturais e Estudos Ambientais, Rio de Janeiro, 124p.

Windisch, P. G. \& Prado, J. 1990. Flora da Serra do Cipó, Minas Gerais. Cyatheaceae. Boletim de Botânica da Universidade de São Paulo 12: 7-13.

Windisch, P. G. 1992. Flora da Serra do Cipó, Minas Gerais. Hymenophyllaceae. Boletim de Botânica da Universidade de São Paulo 13: 133-139. 